\title{
Neurosurgical treatment of low-grade cerebellar astrocytoma in children and adolescents: a single consecutive institutional series of 100 patients
}

\author{
Clinical article
}

\author{
Bernt Johan Due-Tønnessen, M.D., Ph.D., ${ }^{1}$ Tryggve Lundar, M.D., Ph.D., ${ }^{1}$ \\ Arild Egge, M.D., Ph.D., ${ }^{1}$ ANd David Scheie, M.D., Ph.D. ${ }^{2}$ \\ Departments of ${ }^{1}$ Neurosurgery and ${ }^{2}$ Pathology, Oslo University Hospital, Oslo, Norway
}

\begin{abstract}
Object. The objective of this study was to delineate the long-term results of surgical treatment of pediatric lowgrade cerebellar astrocytoma.

Methods. One hundred consecutive children and adolescents (0-19 years old) who underwent primary tumor resection for a low-grade cerebellar astrocytoma during the years 1980-2011 were included in this retrospective study on surgical morbidity, mortality rate, academic achievement, and/or work participation. Gross motor function and activities of daily living were scored according to the Barthel Index.

Results. Of the 100 patients, 61 children were in the 1st decade, and 39 were 10-19 years old. The male/female ratio was 1.13:1 (53 males, 47 females). No patients were lost to follow-up. There were no deaths in this series and all 100 patients are currently alive. In 29 patients, the follow-up duration was less than 10 years, in 37 it was between 10 and 19 years, and in 34 it was between 20 and 31 years. The Barthel Index was 100 (normal) in 97 patients, 90 in 2 patients, and 40 in the last patient. A total of 113 tumor resections were performed. Two patients underwent further tumor resection due to MRI-confirmed residual tumor demonstrated on the immediate postoperative MR image (obtained the day after the initial procedure). Furthermore, 9 children underwent repeat tumor resection after MRI-confirmed progressive tumor recurrence up to 10 years after the initial operation. Two of these patients also underwent a third resection, without subsequent radiation therapy, and have experienced 8 and 12 years of tumorfree follow-up thereafter, respectively. A total of $15 \%$ of the patients required treatment for persistent hydrocephalus.

Conclusions. Low-grade cerebellar astrocytoma is a surgical disease, in need of long-term follow-up, but with excellent long-term results. Nine percent of the children in this study underwent repeated surgery due to progressive tumor recurrence, and $15 \%$ were treated for persistent hydrocephalus.

(http://thejns.org/doi/abs/10.3171/2012.11 PEDS12265)
\end{abstract}

$\begin{array}{lcccc}\text { KEY WoRDS } & \bullet & \text { low-grade cerebellar astrocytoma } & \bullet & \text { long-term results } \\ \text { hydrocephalus } & \bullet \quad & \text { suboccipital craniotomy } & \bullet & \text { oncology }\end{array}$

$\mathrm{L}$ OW-GRADE cerebellar astrocytoma is one of the most common pediatric brain tumors. It has been known for more than 80 years as a tumor with good longterm results after resection, since Cushing presented his results in 1931.6 Although later studies have confirmed good long-term results and reduced surgical mortality, there have been some controversies with regard to postoperative treatment in cases of incomplete resection and/ or recurrent tumor. ${ }^{4}$ This report summarizes our experience in 100 consecutive patients who underwent primary operations for low-grade cerebellar astrocytoma over a period of 31 years.

\section{Methods}

Study Population

We retrospectively analyzed a nonselected cohort consisting of 100 patients who were 19 years of age or younger and who underwent primary resection for a low-grade cerebellar astrocytoma during the years 1980-2011 in the Department of Neurosurgery, The National Hospital, Oslo, Norway. The cases were collected from surgical protocols of the relevant time period. Histological evaluation of the lesions confirmed that they were low-grade astrocytomas. This series includes all cases of low-grade cerebellar astro- 
cytomas in this period, as well as cases in which the brachium pontis was partially affected by the tumor, and those in which the floor of the fourth ventricle was slightly affected by the tumor, but not cases with intrinsic brainstem tumors with or without exophytic extension. Three patients had neurofibromatosis Type 1 and 1 patient had tuberous sclerosis. The data in the case records included sex, age at the time of primary tumor resection, and treatment of eventual persistent hydrocephalus. The data on persistent hydrocephalus were analyzed using the Fisher exact test. For patients who had been followed in other neurosurgical units, information was also obtained from the relevant departments. Scholastic outcome was simplified into normal versus special schooling, and employment attendance simplified as open (standard jobs), sheltered (jobs given to those with disabilities), or no work.

\section{Tumor Neuroimaging}

From 1987, when MRI became available, tumors were visualized on preoperative MRI and repeat MRI was introduced in the follow-up evaluation. The aim of the surgical procedure was gross-total resection, and the degree of resection was evaluated by immediate postoperative MR images, most often during the same anesthetic procedure. During the years 1993-1996, after some experience with MRI in the management of these patients, our practice changed to performing a suboccipital craniotomy instead of a craniectomy during these procedures. In total, 37 patients underwent a craniectomy in the first part of this series, while 63 patients were operated on via a craniotomy thereafter.

\section{Assessment of Functional Status}

The Barthel Index score is a well-established and validated scale that uses 10 variables to measure performance in basic activities of daily living, primarily related to personal care and mobility. ${ }^{14}$ Scores range from 0 to 100 , and a higher score denotes greater independence. The purpose of using this measure was to assess functional status and illustrate eventual differences among subgroups within our cohort.

\section{Results}

The age of the children at primary surgery is illustrated in Fig. 1. Sixty-one patients were in the 1st decade and 39 were in the 2 nd decade. The median age of the study population was 8 years. The male/female ratio was 1.13:1 (53 males, 47 females).

There were no surgical deaths in this series. All patients are alive, and their current age distribution is $2-48$ years. No patients were lost to follow-up, and the followup period was 0-9 years in 29 patients, 10-19 years in 37, and 20-31 years in 34 patients (Fig. 2).

Although there was no surgical mortality in this series, 2 children demonstrated cerebellar mutism (mute and unresponsive) in the immediate postoperative period. Both of these children underwent operations for extensive midline tumors. The first patient, a 5-year-old boy, woke up 3 weeks after the operation and recovered rapidly

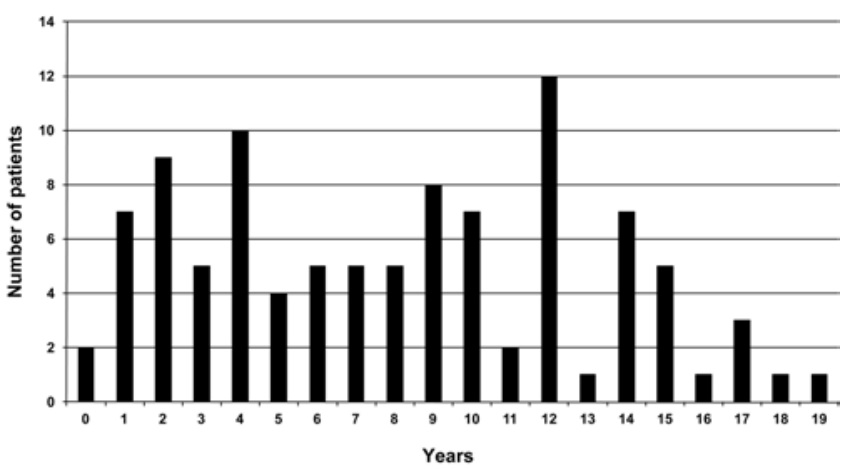

FIG. 1. Age distribution of the 100 patients at the primary operation for low-grade cerebellar astrocytoma.

thereafter. The second patient, a 7-year-old girl, recovered slowly and gradually. Five years after the operation she is clearly ataxic, but otherwise quite well.

\section{Motor Function and Activities of Daily Living}

Ninety-seven patients had a normal Barthel Index of 100 . There was only 1 significantly poor result on the Barthel Index. A 12-year-old boy was operated on in 1985 for a cerebellar astrocytoma. In 1991 he underwent a second resection because of a large recurrent tumor, at that point partially affecting the brainstem. Postoperatively he experienced a left-sided hemiplegia, right-sided facial palsy, ataxia, and partial swallowing problems. He also received a shunt for hydrocephalus. During a shunt failure episode in 1999, he underwent a ventriculocisternostomy and his ventriculoperitoneal shunt was explanted. He is wheelchair-dependent and has a Barthel Index of 40.

Two other patients had a Barthel Index of 90. One of the patients is partly dependent on a gastric tube, and the other has a somewhat reduced function after a serious hydrocephalic episode.

\section{School, Education, and Work Outcomes}

Forty-one patients were younger than 20 years, and all followed a regular school program. Eighteen patients were 20-24 years old. Fifteen of these patients were students or in regular work, while 3 were outside the labor market (unemployed). One of these 3 patients is autistic, as before the operation, and 2 others have severe epilepsy, most probably not related to the cerebellar tumor disease or operative treatment.

The remaining 41 patients were $25-48$ years old.

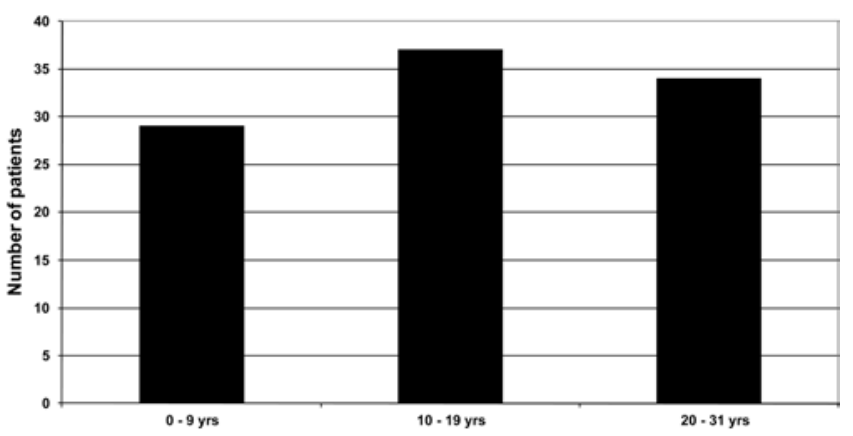

FIG. 2. Distribution of the follow-up duration among the 100 patients. 
Apart from the patient with a Barthel Index of 40, 2 others were unemployed at least partially due to other medical problems, while the rest were well functioning, in partial or full-time work, or home with small children.

\section{Management of Hydrocephalus}

In 90 of these patients hydrocephalus was present, often rapidly progressive, with supratentorial ventricular enlargement on CT or MRI, as well as clinical signs of increased intracranial pressure. One patient underwent a ventriculocisternostomy in advance of the tumor resection, and in 3 children the posterior part of the lateral ventricle was drained by a ventricular catheter in the early phase of the operative procedure to reduce the infratentorial pressure. In the other cases, however, intraoperative CSF drainage from the posterior fossa cisterns was sufficient to control the pressure problem.

Systematic use of methylprednisolone before, during, and after the operation often reversed the clinical signs of raised intracranial pressure before the operative procedure. In 75 of these children, the hydrocephalic problem resolved after the tumor resection. In 15 patients, however, the problem persisted in terms of CSF leakage or reappearance of signs of increased intracranial pressure. In summary, 15 patients experienced a persistent hydrocephalic problem, which was treated using a shunt system. One of these 15 patients first underwent a ventriculocisternostomy procedure, which did not sufficiently resolve the problem. We believe that all 15 cases with persistent hydrocephalus are shunt dependent, and 4 of them have experienced 1 or more episodes of shunt failure that required shunt revision. In 1 of these cases, a ventriculocisternostomy successfully resolved the problem.

During the years 1993-1996 our operative technique was changed from a suboccipital craniectomy to a craniotomy. Ten of the 37 patients who underwent a craniectomy experienced persistent hydrocephalus (28\%), whereas only $5(8 \%)$ of 63 patients in the craniotomy group underwent long-term hydrocephalus treatment $(\mathrm{p}<0.01$, Fisher exact test). Other changes in the management of patients over time could also explain this difference. Four of the 9 patients who underwent repeat tumor resection required hydrocephalus treatment.

\section{Surgical Results}

All 100 of these children underwent a primary tumor resection for a low-grade cerebellar astrocytoma. Three tumors were described as WHO Grade II (fibrillary astrocytomas), 3 were astrocytomas of uncertain type, while the rest were WHO Grade I (pilocytic astrocytomas). In the 1st decade (the 1980s), pre- and postoperative visualization was mostly conducted using CT scans of the quality for that period. Gross-total resection was the aim of the operative procedure, but judgment of the completeness of the resection was also based on the surgeon's description.

In a few children the surgeon deliberately left some tumor tissue unresected due to slight involvement of the brainstem (brachium pontis, floor of the fourth ventricle). In 4 of 32 patients who underwent operations in the 1980s, a subtotal resection was described and postop- erative radiation was administered to the posterior fossa. The ages of these 4 children were $4,7,8$, and 14 years at the time of radiation treatment. The 4-year-old girl developed pituitary deficiency and received pituitary hormone supplementation. She is now 30 years old and performing part-time work.

During the last 23 years no patients have received postoperative radiation therapy, but have been followed clinically and using serial MRI. Most of the patients have demonstrated no residual or recurrent tumor disease on MRI. In a number of cases, however, there was variable (irregular) attenuation within the resection cavity border, but without contrast enhancement. ${ }^{18}$ These cases have proved to be stable over time and without signs of progressive tumor recurrence.

A total of 113 tumor resections were performed. In cases with primary proven residual tumor, further operative resection was performed. This was the case in 2 children in whom the immediate postoperative MRI demonstrated unexpected contrast-enhanced residual tumor, and further resection was undertaken on the next day. In 9 other patients the serial follow-up MRI demonstrated progressive residual or recurrent tumor growth, leading to a second tumor resection, ranging from 3 months to 10 years after the initial procedure (Table 1). Among these patients was a 2-year-old girl with a dramatic tumor recurrence after only 14 weeks. This patient received postoperative chemotherapy (low-grade glioma protocol) for a year after the second operation, and has been doing quite well during the subsequent 7-year follow-up. She is the only child who has been administered chemotherapy in this series.

Among the 9 children who underwent a second resection, 2 experienced a second tumor recurrence. Each of these patients underwent a third tumor resection, 14 and 18 months after the second operation, respectively. The follow-up results in these 2 patients have been uneventful for another 8 and 12 years, respectively (Table 1).

Three of the patients have neurofibromatosis Type 1 . Two of them were among the children who underwent further resection due to progressive recurrence, and 1 also underwent a third resection.

TABLE 1: Patients undergoing repeated tumor resection for low-grade cerebellar astrocytoma

\begin{tabular}{ccc}
\hline Age/Sex at Primary Op & $\begin{array}{c}\text { Time to 2nd } \\
\text { Resection }\end{array}$ & $\begin{array}{c}\text { Time to 3rd Resection } \\
\text { (after 2nd resection) }\end{array}$ \\
\hline $10 \mathrm{mos} / \mathrm{F}$ & $3 \mathrm{mos}$ & \\
$11 \mathrm{mos} / \mathrm{F}$ & $2 \mathrm{yrs}$ & \\
$1 \mathrm{yr} / \mathrm{M}$ & $2 \mathrm{yrs}$ & \\
$1.5 \mathrm{yrs} / \mathrm{F}$ & $10 \mathrm{yrs}$ & \\
$2 \mathrm{yrs} / \mathrm{F}$ & $5 \mathrm{yrs}$ & \\
$4 \mathrm{yrs} / \mathrm{M}$ & $6 \mathrm{yrs}$ & \\
$12 \mathrm{yrs} / \mathrm{M}$ & $6 \mathrm{yrs}$ & $14 \mathrm{mos}$ \\
$12 \mathrm{yrs} / \mathrm{M}$ & $3 \mathrm{yrs}$ & $18 \mathrm{mos}$ \\
$16 \mathrm{yrs} / \mathrm{F}$ & $2 \mathrm{yrs}$ & \\
\hline
\end{tabular}




\section{B. J. Due-Tønnessen et al.}

\section{Discussion}

Cerebellar low-grade astrocytomas have been encountered to be a distinct pediatric tumor with excellent results after resection. Cushing ${ }^{6}$ presented good clinical results with these tumors more than 80 years ago. During the 1970s, operative mortality fell subsequently, most probably due to improvements in operative technique (such as use of the microscope and the ultrasound aspirator), CT scanning, neuroanesthesia, and neurointensive care. Gjerris and Klinken ${ }^{10}$ reported a 25 -year survival rate for cerebellar astrocytoma (WHO Grade I) of $94 \%$ for children operated on between 1935 and 1959 and who survived the operative period. The 25 -year survival was only $38 \%$ for those with diffuse astrocytoma (WHO Grade II). The same institution in 2003 claimed that the prognostic value of the subclassification may not be valid. ${ }^{3}$ In our series, the number of nonpilocytic tumors was so small that it does not provide information on the impact of this category.

Heiskanen and Lehtosalo ${ }^{11,12}$ reported a 5\% surgical mortality rate in 55 cerebellar astrocytomas operated on between 1968 and 1982. Among the survivors there was 100\% 5-year survival and 97\% 10-year long-term survival. Three patients demonstrated recurrent tumor and underwent repeat resection, but 2 later died of their tumors.

Abdollahzadeh and coworkers ${ }^{1}$ reported on a series of 66 children operated on for benign cerebellar astrocytomas between 1980 and 1992 without perioperative or postoperative mortality. All patients survived the followup period of 2-12 years. Five patients with incomplete primary resections underwent $1-3$ repeat resections due to tumor progression.

It has been argued that the prognosis of low-grade cerebellar astrocytoma in children younger than 5 years of age may be less favorable., ${ }^{9,13,22}$ Several authors who discuss the impact of age on prognosis, however, refer to series that include infratentorial as well as supratentorial low-grade astrocytomas. In our series, 32 children were $0-4$ years old at the primary surgery. These patients have recovered very well, despite the fact that 6 have undergone repeated resection for recurrent progressive disease. They are now without residual tumor on MRI, and the follow-up duration after the second resection ranged from 5 to 17 years.

Kibirige et al. ${ }^{13}$ claim that the prognosis for children operated on in the first years of life may be even less favorable. Their series, however, included a large proportion of cases operated on before the CT era, and it is difficult to interpret if this tendency could be caused by a lack of modern operative facilities. Nine of our patients were younger than 2 years, and no less than 4 underwent a second resection for recurrent tumor, but they are all doing well after an uneventful follow-up period of 5-17 years after the second resection.

This single-institution consecutive series included 100 cases without any operative mortality, and covers a period of 31 years. It is well known that the biological behavior of these tumors may vary, even if the results are generally good in this group. In the time before MRI of the posterior fossa became available (1987), evaluation of residual or recurrent tumor problems was more difficult. In this series, 4 of 32 patients who underwent primary operations in the 1980s were given postoperative radiation to the tumor field, based on known or anticipated residual tumor. Based on details on immediate postoperative and follow-up MR images, none of the remaining 68 patients received postoperative radiation. This shift in policy was also influenced by our experience with postoperative radiation treatment of children with posterior fossa medulloblastomas after 1974, with subsequent improvement in survival, but with considerable incidence of treatment sequelae.

Therefore, our strategy has been to perform repeat resection when indicated. Apart from 2 patients who underwent further resection on the next day due to unexpected residual tumor, 9 patients underwent a second tumor resection due to progressive residual or recurrent tumor. Only once was this second operation performed early: in the young girl with dramatic recurrence after only 3 months. This patient was the only one who received chemotherapy for a year after the second resection. The other repeat operations were performed up to 10 years after the initial procedure.

No patient currently has progressive disease, but several are in an early follow-up period. A few have a stable residual tumor, in which further resection has been withheld due to localization in the brachium pontis. In other patients, the wall of the resection cavity has characteristics that may include small amounts of nonenhancing tumor, but where repeated imaging over time does not indicate progressive disease.

As discussed previously, we have also encountered some cases in which follow-up images have demonstrated contrast enhancement that has diminished over time or disappeared, and where spontaneous tumor regression may have taken place. ${ }^{8}$ Several authors ${ }^{2,20}$ have pointed to such spontaneous regression of residual tumor, and it is well known that incomplete resection may be sufficient to obtain long-term control of disease.$^{17}$ It is therefore our policy to wait for residual tumor growth before a second resection is considered, unless it is found on the immediate postoperative MR image and appears easily resectable. Others consider radiation therapy for recurrent tumor as an alternative to a second resection, and it should be considered if a repeat resection involves a considerable risk with respect to novel neurological deficits. ${ }^{19}$ Campbell and Pollack ${ }^{5}$ defer radiation therapy until there is evidence of progressive disease that is surgically unresectable.

Another topic for discussion is the need for long-term follow-up in these patients. In this series, a second resection was performed in $9 \%$ of the patients, up to 10 years after the primary treatment. In a patient operated on prior to this series, we performed a second resection after 21 years, and benign recurrence after radical removal of pilocytic cerebellar astrocytoma has been reported up to 36 years. ${ }^{16}$ Whereas very late recurrence after gross-total resection may be very rare, and the need for prolonged MRI follow-up may be a matter of dispute, late recurrences do occur. ${ }^{16}$ Ogiwara and coworkers ${ }^{15}$ indicate that a follow-up period of 8-10 years is appropriate in patients in whom a complete resection has been performed. Sut- 
ton et al. ${ }^{21}$ claim that children with completely resected cerebellar astrocytomas do not benefit from routine surveillance, but that surveillance is of benefit in those who may demonstrate subtotal resection on immediate postoperative MRI. The question of the degree of radical tumor resection on such MR images may, however, be a more difficult topic than a question of yes or no. ${ }^{18}$ Our policy has therefore been to conduct long-term follow-up using MRI. If the 5-year image appears to show no recurrence, 5- to 10-year intervals may be appropriate. In the subgroup of patients with persistent hydrocephalus, the MR images provide valuable additional information.

The incidence of persistent hydrocephalus after posterior fossa tumor surgery varies among different patient series and has been related to the tumor type, with less persistent hydrocephalus shown in low-grade astrocytoma cases. In a previous study covering 1990 to 2003, we found no difference in persistent hydrocephalus between the craniectomy and craniotomy groups. ${ }^{8}$ In this series, with a larger number of craniotomy cases, the incidence of individuals with shunts is lower in the later part of the study period. This difference could be associated with the introduction of craniotomy instead of craniectomy, or other changes in the management of patients over time.

\section{Conclusions}

Low-grade cerebellar astrocytoma is a disease with very rewarding long-term prognosis. Resection and even repeated surgery is generally well tolerated. In cases in which the brainstem was slightly affected, subtotal resection appeared to control the disease in most cases. Fifteen percent of the patients received shunts due to persistent hydrocephalus and $9 \%$ underwent repeated resection.

\section{Disclosure}

The authors report no conflict of interest concerning the materials or methods used in this study or the findings specified in this paper.

Author contributions to the study and manuscript preparation include the following. Conception and design: all authors. Acquisition of data: Lundar, Due-Tønnessen. Analysis and interpretation of data: all authors. Drafting the article: Lundar, DueTønnessen. Critically revising the article: all authors. Reviewed submitted version of manuscript: all authors. Approved the final version of the manuscript on behalf of all authors: Lundar.

\section{References}

1. Abdollahzadeh M, Hoffman HJ, Blazer SI, Becker LE, Humphreys RP, Drake JM, et al: Benign cerebellar astrocytoma in childhood: experience at the Hospital for Sick Children 19801992. Childs Nerv Syst 10:380-383, 1994

2. Benesch M, Eder HG, Sovinz P, Raith J, Lackner H, Moser A, et al: Residual or recurrent cerebellar low-grade glioma in children after tumor resection: is re-treatment needed? A single center experience from 1983 to 2003. Pediatr Neurosurg 42:159-164, 2006

3. Bernhardtsen T, Laursen H, Bojsen-Møller M, Gjerris F: Subclassification of low-grade cerebellar astrocytoma: is it clinically meaningful? Childs Nerv Syst 19:729-735, 2003

4. Bucy PC, Thieman PW: Astrocytomas of the cerebellum. A study of a series of patients operated upon over 28 years ago. Arch Neurol 18:14-19, 1968

5. Campbell JW, Pollack IF: Cerebellar astrocytomas in children. J Neurooncol 28:223-231, 1996

6. Cushing H: Experiences with the cerebellar astrocytomas: a critical review of seventy-six cases. Surg Gynecol Obstet 52: 129-204, 1931

7. Due-Tønnessen BJ, Helseth E: Management of hydrocephalus in children with posterior fossa tumors: role of tumor surgery. Pediatr Neurosurg 43:92-96, 2007

8. Due-Tønnessen BJ, Helseth E, Scheie D, Skullerud K, Aamodt G, Lundar T: Long-term outcome after resection of benign cerebellar astrocytomas in children and young adults (0-19 years): report of 110 consecutive cases. Pediatr Neurosurg 37:71-80, 2002

9. Gajjar A, Sanford RA, Heideman R, Jenkins JJ, Walter A, Li Y, et al: Low-grade astrocytoma: a decade of experience at St. Jude Children's Research Hospital. J Clin Oncol 15:27922799, 1997

10. Gjerris F, Klinken L: Long-term prognosis in children with benign cerebellar astrocytoma. J Neurosurg 49:179-184, 1978

11. Heiskanen O: Intracranial tumors of children. Childs Brain 3:69-78, 1977

12. Heiskanen O, Lehtosalo J: Surgery of cerebellar astrocytomas, ependymomas and medulloblastomas in children. Acta Neurochir (Wien) 78:1-3, 1985

13. Kibirige MS, Birch JM, Campbell RH, Gattamaneni HR, Blair V: A review of astrocytoma in childhood. Pediatr Hematol Oncol 6:319-329, 1989

14. Mahoney FI, Barthel DW: The Barthel Index. Md State Med J 14:61-65, 1965

15. Ogiwara H, Bowman RM, Tomita T: Long-term follow-up of pediatric benign cerebellar astrocytomas. Neurosurgery 70: 40-48, 2012

16. Pagni CA, Giordana MT, Canavero S: Benign recurrence of a pilocytic cerebellar astrocytoma 36 years after radical removal: case report. Neurosurgery 28:606-609, 1991

17. Palma L, Celli P, Mariottini A: Long-term follow-up of childhood cerebellar astrocytomas after incomplete resection with particular reference to arrested growth or spontaneous tumour regression. Acta Neurochir (Wien) 146:581-588, 2004

18. Rollins NK, Nisen P, Shapiro KN: The use of early postoperative MR in detecting residual juvenile cerebellar pilocytic astrocytoma. AJNR Am J Neuroradiol 19:151-156, 1998

19. Saunders DE, Phipps KP, Wade AM, Hayward RD: Surveillance imaging strategies following surgery and/or radiotheraphy for childhood cerebellar low-grade astrocytoma. J Neurosurg (2 Suppl) 102:172-178, 2005

20. Steinbok P, Poskitt K, Hendson G: Spontaneous regression of cerebellar astrocytoma after subtotal resection. Childs Nerv Syst 22:572-576, 2006

21. Sutton LN, Cnaan A, Klatt L, Zhao H, Zimmerman R, Needle $\mathrm{M}$, et al: Postoperative surveillance imaging in children with cerebellar astrocytomas. J Neurosurg 84:721-725, 1996

22. Taylor MD, Sanford RA, Boop FA: Cerebellar pilocytic astrocytomas, in Albright AL, Pollack IF, Adelson PD (eds): Principles and Practice of Pediatric Neurosurgery, ed 2. New York: Thieme, 2007, pp 655-667

Manuscript submitted May 18, 2012.

Accepted November 5, 2012.

Please include this information when citing this paper: published online December 14, 2012; DOI: 10.3171/2012.11.PEDS12265.

Address correspondence to: Tryggve Lundar, M.D., Department of Neurosurgery, Oslo University Hospital, Postboks 4950 Nydalen, 0424 Oslo, Norway.email: tryggve.lundar@ous-hf.no. 